\title{
Neural stem/precursor cells dynamically change their epigenetic landscape to differentially respond to $B M P$ signaling for fate switching during brain development
}

\author{
Sayako Katada, ${ }^{1}$ Jun Takouda, ${ }^{1}$ Takumi Nakagawa, ${ }^{1}$ Mizuki Honda, ${ }^{1}$ Katsuhide Igarashi, ${ }^{2}$ \\ Takuya Imamura, ${ }^{3}$ Yasuyuki Ohkawa, ${ }^{4}$ Shoko Sato, ${ }^{5}$ Hitoshi Kurumizaka, ${ }^{5}$ and Kinichi Nakashima ${ }^{1}$ \\ ${ }^{1}$ Department of Stem Cell Biology and Medicine, Graduate School of Medical Sciences, Kyushu University, Higashi-ku, Fukuoka \\ 812-8582, Japan; ${ }^{2}$ Institute for Advanced Life Sciences, Hoshi University, Shinagawa-ku, Tokyo 142-8501, Japan; ${ }^{3}$ Program of \\ Biomedical Science, Graduate School of Integrated Sciences for Life, Hiroshima University, Higashi-Hiroshima, Hiroshima \\ 739-8526, Japan; ${ }^{4}$ Division of Transcriptomics, Medical Institute of Bioregulation, Kyushu University, Higashi-ku, Fukuoka \\ 812-8582, Japan; ${ }^{5}$ Laboratory of Chromatin Structure and Function, Institute for Quantitative Biosciences, The University of \\ Tokyo, Bunkyo-ku, Tokyo 113-0032, Japan
}

During neocortical development, tight regulation of neurogenesis-to-astrogenesis switching of neural precursor cells (NPCs) is critical to generate a balanced number of each neural cell type for proper brain functions. Accumulating evidence indicates that a complex array of epigenetic modifications and the availability of extracellular factors control the timing of neuronal and astrocytic differentiation. However, our understanding of NPC fate regulation is still far from complete. Bone morphogenetic proteins (BMPs) are renowned as cytokines that induce astrogenesis of gliogenic late-gestational NPCs. They also promote neurogenesis of mid-gestational NPCs, although the underlying mechanisms remain elusive. By performing multiple genome-wide analyses, we demonstrate that Smads, transcription factors that act downstream from BMP signaling, target dramatically different genomic regions in neurogenic and gliogenic NPCs. We found that histone H3K27 trimethylation and DNA methylation around Smadbinding sites change rapidly as gestation proceeds, strongly associated with the alteration of accessibility of Smads to their target binding sites. Furthermore, we identified two lineage-specific Smad-interacting partners-Sox11 for neurogenic and Sox 8 for astrocytic differentiation - that further ensure Smad-regulated fate-specific gene induction. Our findings illuminate an exquisite regulation of NPC property change mediated by the interplay between cell-extrinsic cues and -intrinsic epigenetic programs during cortical development.

[Keywords: neural stem/precursor cell; differentiation; bone morphogenetic protein; Smad; neuron; astrocyte; epigenetic modifications; chromatin accessibility]

Supplemental material is available for this article.

Received June 29, 2021; revised version accepted September 28, 2021.

Neural stem and precursor cells (NPCs, used here collectively) are defined as self-renewable and multipotent cells that give rise to neurons and two types of glial cells: astrocytes and oligodendrocytes (Temple 2001). However, NPCs do not have the ability to differentiate into the three cell types at the earliest stages of development. In the developing cerebral cortex, NPCs first undergo repeated symmetric division to expand their progenitor pools and

Corresponding authors: kin1@scb.med.kyushu-u.ac.jp, sakatada@scb.med.kyushu-u.ac.jp

Article published online ahead of print. Article and publication date are online at http://www.genesdev.org/cgi/doi/10.1101/gad.348797.121. Freely available online through the Genes \& Development Open Access option. then differentiate first into neurons, and later into astrocytes and oligodendrocytes (Paridaen and Huttner 2014). The precise switching of differentiation competence from neurogenic to gliogenic is especially important to generate proper numbers of neural cells for the appropriate brain functions (Miller and Gauthier 2007). In this context, several extracellular factors secreted from cells residing adjacent to NPCs have been shown to influence the neuron-astrocyte differentiation switch of NPCs

(C) 2021 Katada et al. This article, published in Genes \& Development, is available under a Creative Commons License (Attribution-NonCommercial 4.0 International), as described at http://creativecommons.org/licenses/by-nc/4.0/. 
(Barnabé-Heider et al. 2005; Kawamura et al. 2017; Duong et al. 2019). However, it has also been suggested that the fate switching is cell-intrinsically programmed, since, for instance, cultured NPCs derived from the embryonic forebrain, as well as embryonic stem cell-derived NPCs, follow the same sequence of cell generation as is observed in the developing brain (Shen et al. 2006; Eiraku et al. 2008; Gaspard et al. 2008; Renner et al. 2017). Therefore, it has become generally accepted that extracellular cues and intracellular programs cooperatively play critical roles in the control of this fate switching (Rowitch and Kriegstein 2010; Nakagawa et al. 2020).

Evidence is accumulating that epigenetic changes around the key regulatory regions of both neuronal and astrocytic genes are deeply implicated in the cell fate switch (Albert and Huttner 2018; Nakagawa et al. 2020). The repressive histone $\mathrm{H} 3$ lysine 27 trimethylation (H3K27me3) around Neurog1 is increased in late-gestational NPCs, leading to the suppression of the gene (Hirabayashi et al. 2009). Since Neurog 1 is one of the determinants for generation of excitatory neurons in the neocortex (Fode et al. 2000), H3K27me3 of Neurog1 by Polycomb group (PcG) proteins reduces neurogenic potential in late-gestational NPCs. On the other hand, another repressive modification, DNA methylation of astrocytic genes, such as glial fibrillary acidic protein (Gfap) and S100 calcium-binding protein $B(S 100 b)$, is high in mid-gestational NPCs, repressing astrocytic differentiation (Takizawa et al. 2001; Namihira et al. 2009). The maintenance DNA methyltransferase 1 (Dnmt1) has been shown to associate with these astrocytic genes to maintain their methylated DNA status; however, the expression of nuclear factor IA (Nfia), a transcription factor important for astrocytic differentiation (Shu et al. 2003), is gradually up-regulated as development progresses and induces the eviction of Dnmt 1 from astrocytic genes, resulting in the acquisition of gliogenic competence by NPCs in the later stage (Namihira et al. 2009; Sanosaka et al. 2017). Nevertheless, the mechanisms of how NPC fate choice is switched remain to be fully elucidated.

Bone morphogenetic proteins (BMPs) are a group of cytokines that are commonly used to induce astrocytic differentiation of NPCs while inhibiting neuronal differentiation (Nakashima et al. 2001; Bonaguidi et al. 2005), and their downstream signals are well characterized (Cole et al. 2016). Canonically, BMPs induce phosphorylation of receptor-regulated (R)-Smads (Smad1/5/9), which then undergo complex formation with the common mediator Smad (Smad4); these complexes translocate into the nucleus and bind cis-regulatory elements (CREs) of their target genes to either activate or repress transcription (Miyazono et al. 2007). Although the astrocyte-inducing activity of BMPs against late-gestational NPCs is widely reported, BMPs are additionally capable of inducing neuronal differentiation of mid-gestational NPCs (Li et al. 1998; Zechner et al. 2007), and we have also confirmed that BMP2 induces neuronal differentiation of mid-gestational NPCs from mouse brains at embryonic day 11 (E11) (Fig. 1A). However, the molecular mechanism of these stage-dependent and fate switch-re- flecting distinct responses to BMPs of NPCs has yet to be fully elucidated.

In the current study, to clarify the mechanisms underlying these distinct effects of BMP2 on developmentally different NPCs, we performed ChIP-seq for activated Smads and RNA-seq analyses of mid-gestational (E11) and lategestational (E14) NPCs before and after BMP2 treatment, and identified genome-wide Smad-binding CREs that are involved in neuronal or astrocytic differentiation of NPCs. Moreover, our ATAC-seq (assay for transposase-accessible chromatin using sequencing) and global analyses of epigenetic modifications around Smad-binding sites yielded the detailed molecular insight that the accessibility of Smads to chromatin collaborates with lineagespecific interactive partners of Smads to control the developmental stage-specific choice of NPC differentiation.

\section{Results}

BMP2 induces neuronal or astrocytic differentiation of NPCs depending on developmental stage

As well as being astrocyte-inducing cytokines, BMPs are also known to inhibit neuronal differentiation of late-gestational NPCs (Nakashima et al. 2001; Kohyama et al. 2010). However, some studies have also demonstrated that BMPs generate neurons in mid-gestational NPCs (Li et al. 1998; Zechner et al. 2007), prompting us to elucidate the molecular mechanism underlying the responsiveness of NPCs to BMPs during cortical development. We first differentiated NPCs derived from mouse brains at E11 (mid-gestation) and E14 (late gestation) for $4 \mathrm{~d}$ in the presence or absence of BMP2, and evaluated the generated cell types. As previously shown, BMP2 enhanced neuronal differentiation of E11 NPCs but induced astrocytic differentiation of E14 NPCs (Fig. 1A). Since a subfraction of cells had already committed to the neuronal lineage at the time when NPCs were prepared from the embryonic cortices, withdrawal of basic fibroblast growth factor (bFGF), a mitogen for NPCs, induced some spontaneous differentiation into Tubb3-positive neurons in both E11 and E14 NPCs (Fig. 1A). However, BMP2 dramatically enhanced neuronal differentiation and almost none of the cells remained positive for Sox2, a marker of NPCs, in E11 NPCs (Supplemental Fig. S1). Conversely, BMP2 clearly inhibited neuronal differentiation and induced astrocytic differentiation in E14 NPCs, in accordance with previous reports (Nakashima et al. 2001; Kohyama et al. 2010). To evaluate this BMP2-induced neuronal or astrocytic gene expression across the genome, we performed RNA-seq analyses of E11 and E14 NPCs before and $24 \mathrm{~h}$ after BMP2 treatment. We observed 1349 or 1449, and 1793 or 1448 significantly up-regulated or down-regulated genes in E11 and E14 NPCs, respectively, in response to BMP2 treatment (Supplemental Fig. S2A,B). Although a heat map of the top 2500 variable genes clearly demonstrated that expression of different sets of genes was increased or reduced between E11 and E14 NPCs after BMP2 stimulation (Fig. 1B), a small group of genes, including well-known BMP target genes (such as Hey1, Id1, and 
A
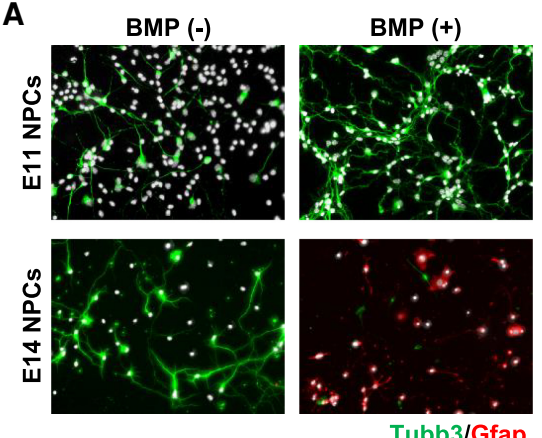

E11 NPCs

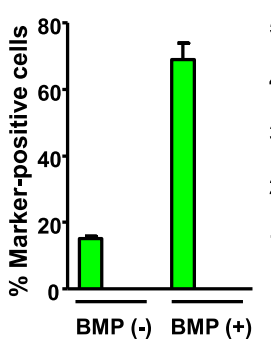

E14 NPCs

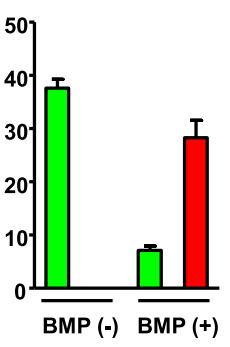

B
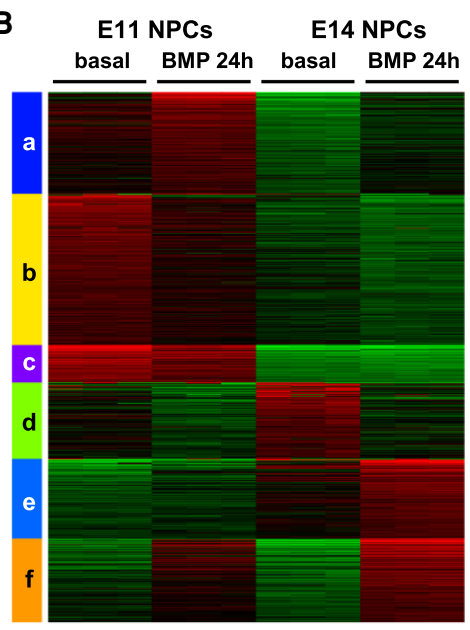

Log2 FC $-4 \begin{array}{lllll}-2 & 0 & 2 & 4\end{array}$ DIx2 Msx1 Msx2 Meurog1 Dlx1 Emx1
Fezf2
Foxp1 Foxp1
Foxp2 Lhro5
Zic1
Otx2
Nkx2.1 Otx2 Zic2 DII1 Sox9
Sox10 Sox10
Gfap Gfap
Nfia
Nfix Nfix
Sox 8 Sox 8 Hey1
Id1 1
ld 2 $\operatorname{ld} 2$
$\operatorname{lnsm} 1$

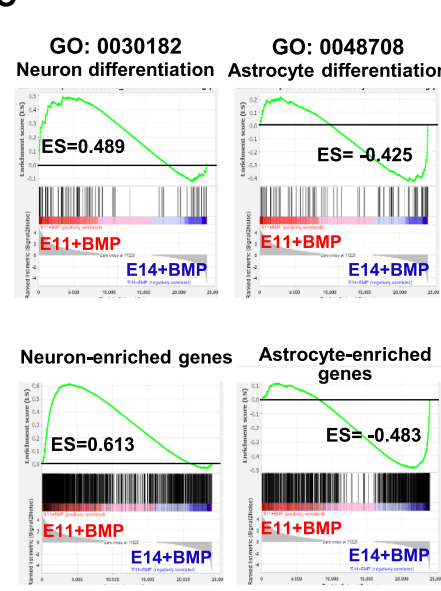

D

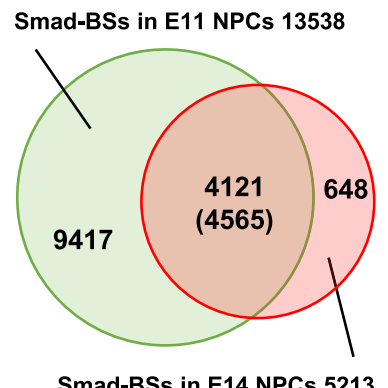

E

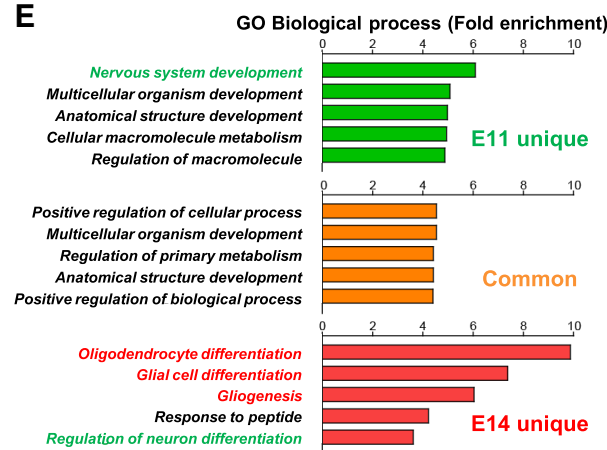

Figure 1. BMP2 induces neuronal and astrocytic differentiation in mid- and late-gestational NPCs, respectively. (A) NPCs derived from E11 or E14 forebrain were differentiated for $4 \mathrm{~d}$ in the presence or absence of BMP2, and immunostained with anti-Tubb3 (green) and anti-Gfap (red) antibodies. (B) RNA-seq was performed on E11- and E14-derived NPCs before and $24 \mathrm{~h}$ after BMP2 treatment. Heat maps for the top 2500 variable genes are shown together with the results of $\mathrm{K}$-means clustering using $k=6$. The colors display the relative gene expression on a $\log _{2}$ scale. Green indicates the lowest, black indicates the intermediate, and red indicates the highest expression. (C) Enrichment score plots for neuron differentiation (GO: 0030182), astrocyte differentiation (GO: 0048708), and neuron- or astrocyteenriched gene sets using BMP2-treated E11 versus E14 NPCs. (D) Venn diagram showing BMP2-induced Smad-BSs in E11 and E14 NPCs identified by ChIP-seq. (E) GO terms (biological process) for E11 unique, E14 unique, and E11/ 14 common Smad-BS-associated genes.
Id2), was commonly up-regulated (Fig. 1B, cluster f; Supplemental Fig. S2C). The heat map also revealed that expression of well-characterized neuronal transcription factors was high in E11 NPCs (Fig. 1B, clusters a-c), and BMP2 treatment further up-regulated a subset of these genes such as Neurog1, Dlx2, and Msx1/2 (Fig. 1B, cluster a; Supplemental Fig. S2C). On the other hand, we noticed that known astrocyte-related genes, such as Gfap, Nfia/x, and Sox8, were predominantly induced in E14 but not in E11 NPCs (Fig. 1B, cluster e; Supplemental Fig. S2C). Gene set enrichment analysis further supported the differential responsiveness of NPCs to BMP2 stimulation, with the gene ontology (GO) terms of neuron differentiation (GO: 0030182) and astrocyte differentiation (GO: 0048708) and with self-assigned gene sets of neuron-enriched (includes 1055 genes) and astrocyte-enriched (in- cludes 720 genes) being enriched in E11 and E14 NPCs, respectively (Fig. 1C). Taken together, these results strongly indicate that BMP2 induces neuronal differentiation of E11 NPCs, and conversely enhances astrocytic differentiation of E14 NPCs.

\section{Smads are recruited to different gene loci in E11} and E14 NPCs

BMP2 transduces signals by binding to type I and II serine/ threonine kinase receptor complexes, which in turn activate canonical signaling via $\mathrm{R}-\mathrm{Smads}(\operatorname{Smad} 1 / 5 / 9)$. These form a complex with Smad4, triggering nuclear translocation and binding to target sequences (Smad-BSs). To identify Smad-BSs across the genomes of E11 and E14 NPCs, we performed ChIP-seq analyses using an antibody 
against phosphorylated-Smad1/5/9 before and $1 \mathrm{~h}$ after BMP2 stimulation. We identified 13,538 and 5213 BMP2-dependent Smad-BSs in E11 and E14 NPCs, respectively, using ChIP samples of the corresponding unstimulated NPCs as a control (Fig. 1D). De novo motif prediction was performed for E11 and E14 Smad-BSs, and at both stages the Smad-binding motif GGCGCC, similar to a previously reported motif (Morikawa et al. 2011; Ramachandran et al. 2018), was prominent (Supplemental Fig. S3A) and was remarkably enriched at the center of actually identified Smad-BSs (Supplemental Fig. S3B), validating the quality of our ChIP-seq experiments. Although the motifs we found were similar between the two developmental stages (Supplemental Fig. S3A), their locations were different: $70 \%$ of the Smad-BSs identified in E11 NPCs were classified as E11-specific (Fig. 1D). These results well reflected the difference between E11 and E14 NPCs in their gene expression responsiveness to BMP2 (Fig. 1B). On the other hand, $\sim 12 \%$ (648 of 5213) of Smad-BSs identified in E14 NPCs were E14specific.

We then associated these three Smad-BS types (i.e., E11 unique, E14 unique, and E11/14 common) with the single nearest gene within $20 \mathrm{~kb}$ from its transcription start site (TSS), and assessed their GO biological process terms using GREAT. As a result, various categories of developmental terms were highlighted in both E11 unique and E11/14 common Smad-BS-associated genes (Fig. 1E). In contrast, terms of glial cell differentiation and gliogenesis were emphasized among the E14 unique Smad-BS-associated genes (Fig. 1E), indicating that Smads dynamically change their binding loci and induce gliogenic differentiation in the transition from mid- to late-gestational NPCs. To evaluate whether binding of Smads to E11 unique, E14 unique, and E11/14 common Smad-BSs is promotive or suppressive for gene expression, the Smad-associated genes were divided into up-regulated $\left(\log _{2} \mathrm{FC}>0.58, q<\right.$ $0.05)$, down-regulated $\left(\log _{2} \mathrm{FC}<-0.58, q<0.05\right)$, and unchanged gene groups based on our RNA-seq data (Fig. 2A). Approximately $83 \%$ of 9417 E11 unique Smad-BSs are located within $20 \mathrm{~kb}$ of a gene TSS, and 2152 and 1718 of these genes were up-regulated and down-regulated, respectively, by BMP2 stimulation (Fig. 2A). Of the 648 E14 unique Smad-BSs, $53 \%$ of them are located within $20 \mathrm{~kb}$ of a TSS, and 136 and 54 of these genes were upregulated and down-regulated, respectively, by BMP2 stimulation (Fig. 2A). Regarding the 4121 E11/14 common Smad-BSs, $78 \%$ were assigned to genes fulfilling the same $20-\mathrm{kb}$ criterion, and the expression of $73 \%$ of them was up-regulated or down-regulated by BMP2 stimulation. Intriguingly, in the E11/14 common Smad-BS-associated genes, we identified a set of genes whose expression is oppositely regulated in E11 and E14 NPCs. For example, Olig1 and Olig2 were up-regulated twofold $24 \mathrm{~h}$ after BMP2 treatment in E11 NPCs, whereas they were downregulated to half in E14 NPCs (Supplemental Fig. S3D). Conversely, BMP2 reduced Hes5 and Hes6 expression in E11 but induced both in E14 NPCs (Supplemental Fig. $\mathrm{S} 3 \mathrm{D})$; such genes were categorized as "either" in E11/14 common Smad-BSs (Fig. 2A). We noticed that regardless of gene induction capacity and direction, the level of Smads recruited did not differ much (Fig. 2B), and we therefore decided to take all Smad-BSs into account in the following analyses.

\section{Chromatin accessibility regulates stage-specific Smad binding}

To dissect how Smads target each locus in a developmental stage-dependent manner, we next analyzed chromatin accessibility of Smad-BSs in E11 and E14 NPCs with ATAC-seq. We identified 62,429 and 77,258 transposaseaccessible regions in E11 and E14 NPCs, respectively (Supplemental Fig. S4A), and most of these regions overlapped at the two developmental stages $194 \%$ for E11, and $76 \%$ for E14). We then evaluated the accessibility of each Smad-BS and found that chromatin openness of E11/14 common Smad-BSs was the same in E11 and E14 NPCs (Fig. 2C). In contrast, chromatin openness of E11 unique Smad-BSs was higher in E11 NPCs, but that of E14 unique Smad-BSs was lower in E11 NPCs (Fig. 2C). Furthermore, we analyzed chromatin accessibility of Smad-BSs reported in another cell lineage (Baik et al. 2016), and found that Smad-bound sites in mouse mesodermal cells were almost completely closed in both E11 and E14 NPCs (Supplemental Fig. S4B). On closer inspection, we found that Smad-BSs in NPCs and mouse mesodermal cells rarely overlap (Supplemental Fig. S4C), indicating that Smads bind to their cognate sites associated with cell type-specific open chromatin.

Next, we focused on Smad-BSs located in close proximity to or within gene bodies and identified several sets of candidate CREs whose accessibility was dramatically different between E11 and E14 NPCs (Fig. 2D; Supplemental Fig. S5A). As exemplified by candidates CRE1 (7 kb upstream of the TSS) and CRE2 (around the TSS) in the Neurog 1 locus, ATAC-seq revealed that these are predominantly much more accessible than their neighboring regions in E11 NPCs, and Smads were recruited to these CREs in an E11-specific manner (Fig. 2D, left). Conversely, remarkable unique ATAC-seq signals were observed in E14 NPCs within the gene body of $P f k f b 3$, encoding a glycolytic enzyme that is highly expressed in astrocytes but not in neurons (Burmistrova et al. 2019), and Smad binding was observed in this region in E14 but not in E11 NPCs (Fig. 2D, middle). Of note, ATAC signals were equivalently observed between E11 and E14 NPCs in E11/14 common Smad-BSs, such as Id2 and Bmpr1a loci (Fig. 2D, right; Supplemental Fig. S5A, right). Collectively, these findings suggest that Smads bind to stage-specific open chromatin regions in NPCs to regulate the expression of target genes.

\section{H3K27me3 and DNA methylation participate in the control of stage-specific Smad binding}

To obtain further mechanistic insight into developmental stage-dependent Smad binding to the genome, we next investigated epigenetic modifications, such as DNA methylation and histone modifications, since these modifications influence chromatin accessibility (Klemm et al. 
A E11 unique 9417 Smad-BSs

\begin{tabular}{|c|c|}
\hline \multicolumn{2}{|c|}{ Gene associated 7828} \\
\hline Up & Down \\
\hline 2152 & Unchanged \\
\hline
\end{tabular}

B E11 unique Smad-BSs

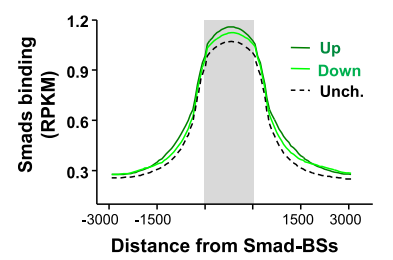

C E11 unique Smad-BSs

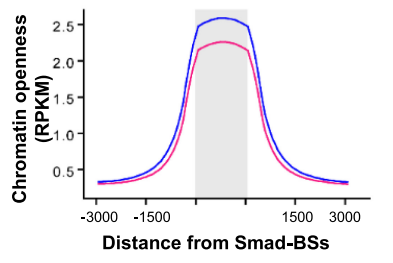

D
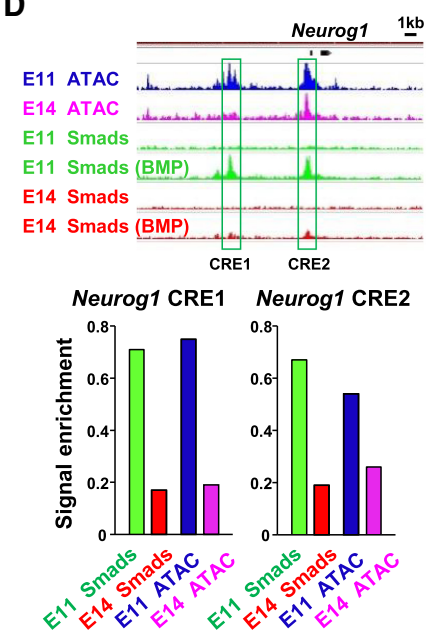

E14 unique 648 Smad-BSs

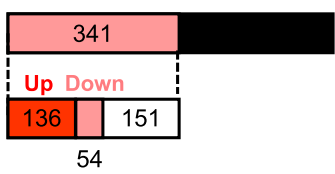

E14 unique Smad-BSs

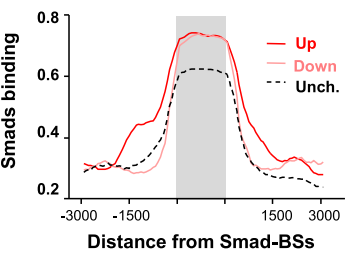

E14 unique Smad-BSs
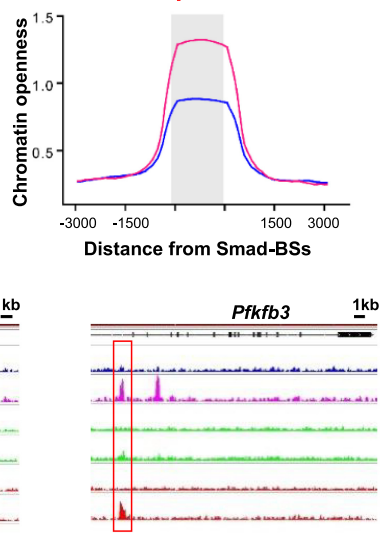

Pfkfb3

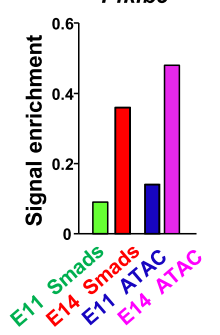

Common 4121 Smad-BSs

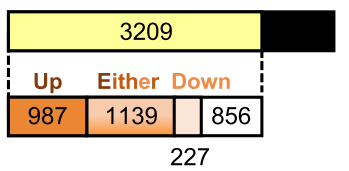

Common Smad-BSs

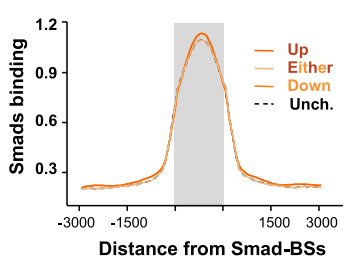

Common Smad-BSs
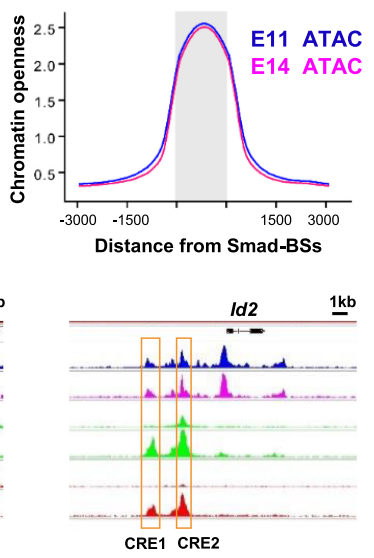

Id2 CRE1

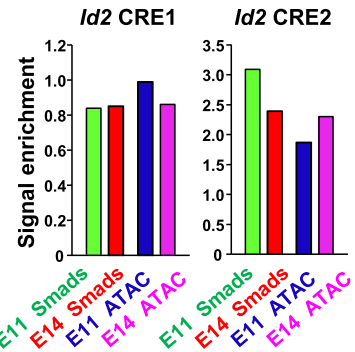

Figure 2. Chromatin accessibility regulates developmental stage-specific Smad binding. $(A)$ Bar graphs showing the number of Smad-BSs located within $20 \mathrm{~kb}$ from the nearest TSS. Associated genes were further classified based on expression changes using RNA-seq data. Besides up-regulated (Up) and down-regulated (Down) genes, common peaks have an "either" category, since some genes exhibit opposite induction in E11 and E14 NPCs following BMP2 stimulation. (B) Plots of Smad ChIP-seq signals around E11 unique, E14 unique, and E11/14 common Smad-BSs. Three types of Smad-BSs were further classified based on their expression change, up-regulated or down-regulated, and either for E11/E14 common Smad-BSs. Smad enrichment profiles of unchanged genes were also plotted (Unch.; dashed lines). (C) Plots of ATAC-seq signals around E11 unique, E14 unique, and E11/14 common Smad-BSs in E11-derived (blue) or E14-derived (magenta) NPCs. $(D)$ Representative genes having E11 unique, E14 unique, and E11/14 common Smad-BSs. Tracks display ATAC-seq signals of basal E11 (blue) and E14 (magenta) NPCs, or Smad ChIP-seq of basal or BMP2-treated E11 (green) and E14 (red) NPCs. Signal enrichment for the boxed regions is expanded in the bar graphs.

2019). We first analyzed the well-characterized repressive histone modification $\mathrm{H} 3 \mathrm{~K} 27 \mathrm{me} 3$ by ChIP-seq. This revealed that the H3K27me3 modification levels of E11/14 common Smad-BSs were low and showed a similar pattern in E11 and E14 NPCs; however, those of E14 unique Smad-BSs were remarkably high in E11 NPCs, but low in E14 NPCs (Fig. 3A; Supplemental Fig. S5A). In contrast to the E14 unique Smad-BSs, H3K27me3 levels of E11 unique Smad-BSs were low in E11 NPCs but high in E14 NPCs, indicating that Smads bound well to their cognate regions with low $\mathrm{H} 3 \mathrm{~K} 27 \mathrm{me} 3$ regardless of the developmental stage (Fig. 3A). Representative H3K27me3 and Smad-binding profiles are shown in Supplemental Figure S5A. For example, in the locus of $D l \times 2$, a transcription factor that participates in the generation of GABAergic interneurons, lower H3K27me3 signals and higher Smad 
binding were detected in BMP2-stimulated E11 NPCs, but the opposite was observed in E14 NPCs. Therefore, it is conceivable that changes in developmental stage-dependent H3K27me3 play a role in defining the binding of Smads to their cognate sites.

DNA methylation levels of each Smad-BS were next evaluated, using our previous whole-genome bisulfite sequencing (WGBS) data from NPCs directly sampled from Sox2-EGFP transgenic mice (Sanosaka et al. 2017). We found that the DNA methylation level of both E11 unique and E11/14 common Smad-BSs is almost zero in E11 NPCs, and this low methylation status is maintained until E18 (Fig. 3B), suggesting that, contrary to H3K27 trimethylation, DNA methylation is not introduced around E11 unique Smad-BSs even in gliogenic NPCs. However, it is notable that DNA methylation levels of E14 unique Smad-BSs are high in E11 NPCs ( $60 \%$ of CpG dinucleotides within Smad-BSs are methylated), but the sites are gradually demethylated and methylation is almost annihilated in E18 NPCs (Fig. 3B). We also examined, individually, the DNA methylation status of -5000 - to +5000-bp genomic fragments centered around all E11 unique, E14 unique, and E11/14 common Smad-BSs, and found that methylation levels are lowest at each Smad-BS in E11, E14, and E18 NPCs (Supplemental Fig. S5B). These results suggest that Smads preferentially bind to accessible target sequences that display low DNA methylation. In previous work, we identified developmental progression-dependent demethylated DNA regions in NPCs /referred to here as reduced differentially methylated regions [RDMRs]). Since demethylation in the R-DMRs is strongly associated with the gliogenic potential of NPCs (Sanosaka et al. 2017), we investigated the overlap between R-DMRs and the ATAC-seq signals identified in the current study. We found that $21.5 \%$ of later-stage unique accessible chromatin regions (E14 unique ATACs) coincided with R-DMRs (Supplemental Fig. S5C), demonstrating that DNA methylation is indeed one of the critical factors regulating chromatin accessibility. Notably, astrocytic differentiation-related Gfap and Nfib are among the representative genes with RDMRs (Sanosaka et al. 2017), and stage-specific accessible regions where Smads bind were observed in these genes in an E14 NPC-specific manner (Fig. 3C). Consequently, these data implicate epigenetic modifications, including DNA methylation and $\mathrm{H} 3 \mathrm{~K} 27 \mathrm{me} 3$, in the determination of binding preference of Smads.

\section{Forced expression of Nfia confers the gliogenic property on mid-gestational NPCs}

We have previously demonstrated that Nfia is involved in DNA demethylation of astrocytic genes, serving as a pivotal transcription factor for the acquisition of gliogenic potential by mid-gestational NPCs (Namihira et al. 2009; Sanosaka et al. 2017). This prompted us to check whether DNA demethylation of Smad-BSs is induced by Nfia. Since the expression level of Nfia is low in mid-gestational NPCs (Sanosaka et al. 2017), we overexpressed Nfia in E11 NPCs and treated the cells with BMP2 to induce differentiation. As shown in Figure 1A, BMP2 gener- ated Tubb3-positive neurons in E11 NPCs, whereas it dramatically produced Gfap-positive astrocytes in Nfiatransduced NPCs (Fig. 3D). We further confirmed the astrocytic differentiation of Nfia-transduced cells by qPCR, revealing that not only Gfap but also several later-stage-specific BMP2-responsive genes, such as Sox8, $S 100 b$, and Clu, were induced specifically in the Nfiaoverexpressing NPCs $24 \mathrm{~h}$ after BMP2 stimulation (Fig. $3 \mathrm{E})$. It is interesting to note that Neurog1 induction was conversely decreased in Nfia-transduced NPCs with BMP2 stimulation (Fig. 3E), clearly demonstrating that a single transcription factor, Nfia, is able to bring about NPC fate switching. The mechanism underlying Neurog1 suppression in Nfia-overexpressing cells is discussed later.

Identification of distinct Sox family members as stagespecific binding partners of Smads

In addition to demonstrating that epigenetic modifications are the key factors affecting chromatin openness and Smad-binding specificity, we also identified distinct factors that further ensure the BMP effects on stage-dependent differentiation of NPCs. When E11 unique Smad-BSs were simply classified into four groups based on their overlap with the ATAC-seq peaks, i.e., E11 unique open, E14 unique open, E11/14 common open, and others (no overlap with the ATAC-seq peaks), we realized that only $7 \%$ coincided with E11 unique open while $78 \%$ were open in both E11 and E14 NPCs (Fig. 4A). Given that about half of the E14 unique Smad-BSs were uniquely open in E14 NPCs (Fig. 4A), although chromatin accessibility is reportedly part of the critical regulatory machinery to control Smad binding, we predicted that other mechanism(s) should exist. Since BMPs function in numerous tissues, many cell type-specific binding partners of Smads have been described (Morikawa et al. 2013), raising the possibility that Smads target their cognate loci with the assistance of stage-specific recruiting partners in NPCs.

To identify developmental stage-specific Smad interactors in NPCs, we re-evaluated the identified Smad-bound sequences. Since we presumed that Smad partners bind to each Smad-BS together with Smads, their binding should also be highlighted in sequences near the Smad-BSs. Therefore, we integrated our ChIP-seq data with the public ChIP-seq database using ChIP-Atlas (https://chip-atlas .org) and asked whether any of these experimentally validated transcription factor-binding sites in neural cell types are enriched in Smad-BSs. We found that multiple SRY-box transcription factor (Sox) family proteins were overrepresented in stage-specific Smad-BSs (Table 1). It appeared that transcription factors $\mathrm{Cbx} 3$ and Sox 4 are exclusively enriched in E11 unique Smad-BSs, and that Nfia, Olig2, Sox2, and Sox9 were outstandingly highlighted in E14 unique Smad-BSs. Previous findings that SoxC family members (Sox4 and Sox11) are critical for neuronal fate commitment (Potzner et al. 2010; Wang et al. 2013), whereas SoxE members (Sox8, Sox9, and Sox10) enhance gliogenesis (Kuhlbrodt et al. 1998; Stolt et al. 2003), motivated us to consider whether a Smad binding partner 

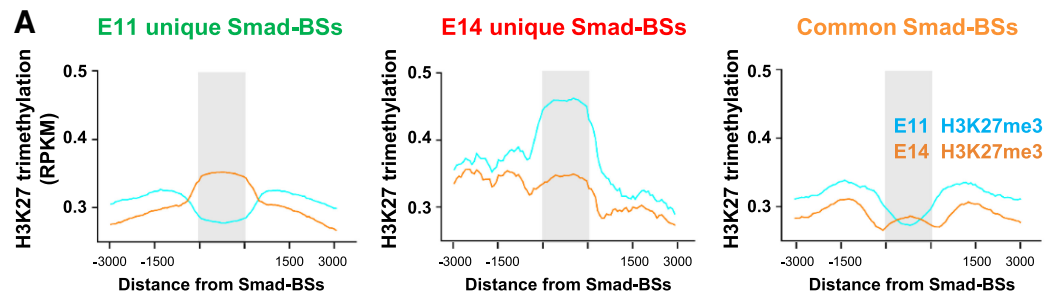

B E11 unique Smad-BSs
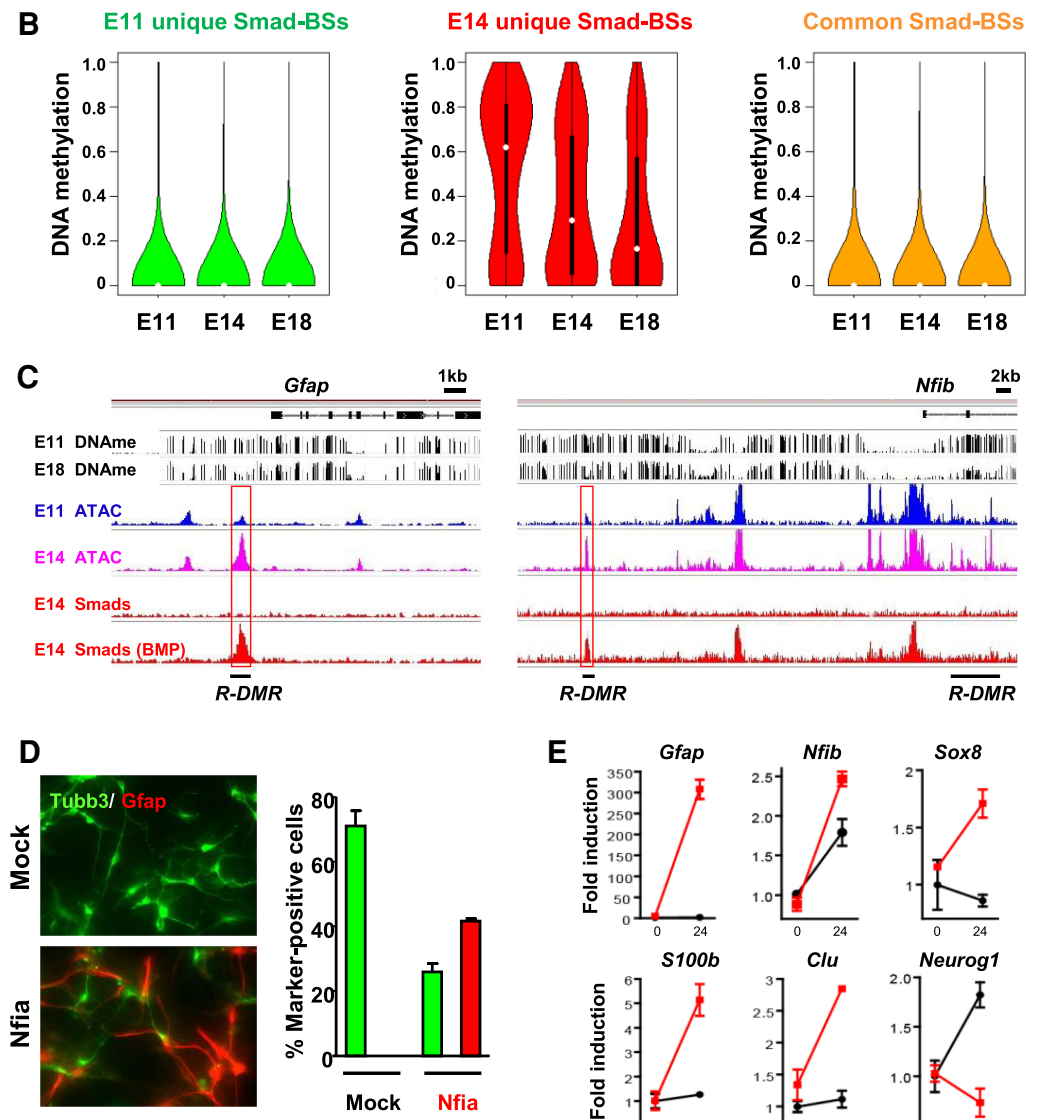

E
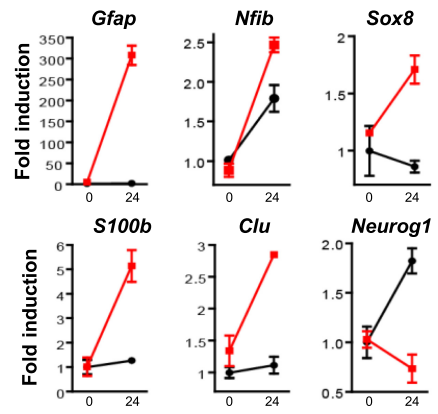

Figure 3. Epigenetic modifications regulate stage-specific Smad binding. (A) Plots of H3K27me3 ChIP-seq signals around E11 unique, E14 unique, and E11/14 common Smad-BSs in E11-derived (blue) or E14-derived (orange) NPCs. (B) Averaged DNA methylation levels of all CpG sites located within E11 unique $(n=60,813)$, E14 unique $(n=1353)$, and E11/14 common $(n=$ $48,924)$ Smad-BSs in NPCs directly isolated from E11, E14, and E18 mouse forebrains. (C) Representative genes having proximal coincident R-DMRs and E14 unique Smad-BSs. Tracks display bisulfite-seq of E11 and E18 NPCs, ATAC-seq of E11 (blue) and E14 (magenta) NPCs, and Smads ChIPseq of untreated or BMP2-treated E14 NPCs (red). Black lines at the bottom show R-DMRs. (D) E11 NPCs were mock-infected or infected with Nfia-expressing virus and cultured for $2 \mathrm{~d}$ in the presence of bFGF, and then stimulated with BMP2 for a further $4 \mathrm{~d}$ to induce differentiation. The cells were stained with antibodies against Tubb3 (green) and Gfap (red). (E) E11 NPCs were mock-infected (black) or infected with Nfia-expressing virus (red) and cultured for $2 \mathrm{~d}$ in the presence of bFGF, and mRNA was isolated before and $24 \mathrm{~h}$ after BMP2 stimulation. Realtime RT-PCR data for selected genes are shown as means $\pm \mathrm{SD}(n=3)$. switch from SoxC to SoxE family members is involved in NPC fate determination.

To evaluate the co-occupancy of SoxC and SoxE family proteins with Smads, we took advantage of prior ChIP-seq data for Sox4 in E14 NPCs (Braccioli et al. 2018) and for Sox9 in glial progenitor cells (Klum et al. 2018). Results in Figure 4B show that $43 \%$ (4848) of Sox 4 peaks overlapped with Smad-BSs identified in either E11 or E14, of which 63\% (3068) were classified as E11 unique SmadBSs. On the other hand, only $10 \%$ (398) of Sox9 peaks coincided with Smad-BSs, but $46 \%$ (185) of them were E14 unique Smad-BSs (Fig. 4B). Density plots of Sox4 and Sox9 enrichment show the highest signals centered in Smad-BSs, with higher Sox4 enrichment in E11 unique than in E14 unique Smad-BSs, and remarkable Sox9 signals centered in E14 unique Smad-BSs (Supplemental Fig. S6A), suggesting that Sox 4 preferentially co-occupies and Sox9 exclusively co-occupies Smad-BSs in E11 and E14 NPCs, respectively. In support of these findings, we observed Sox4 binding in E11 unique Smad-BSs around
Neurog1 and Dlx2, and Sox9 binding in E14 unique Smad-BSs around the Gfap locus (Supplemental Fig. S6B).

Next, we evaluated the interaction of Smads with SoxC and SoxE family proteins, as well as with Sox2 (a SoxB1 member, highlighted as an E14 unique putative Smad partner in Table 1). We found that SoxC proteins (Sox4 and Sox11) and SoxE proteins (Sox8 and Sox9), but not Sox2, interact with Smad components (Smad1 and Smad4) in BMP signal-activated HEK293T cells (Fig. 4C). Immunoprecipitation using anti-phospho-Smad1/5/9 antibody was performed in BMP2-stimulated NPCs and confirmed that Smads interact with Sox11 in E11 NPCs, and with Sox8 in E14 NPCs, in accordance with the endogenous expression levels of each Sox protein (Fig. 4D) and mRNA (Fig. 5A).

Distinct Sox family members oppositely influence stagedependent BMP effects on NPC differentiation

Our finding that Smad binding partners change during NPC progression encouraged us to test whether the 
A

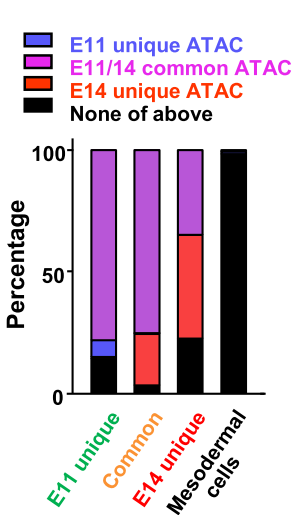

B
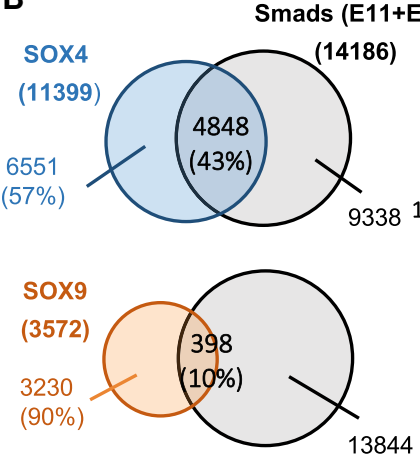

13844

C

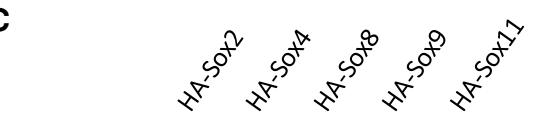

D

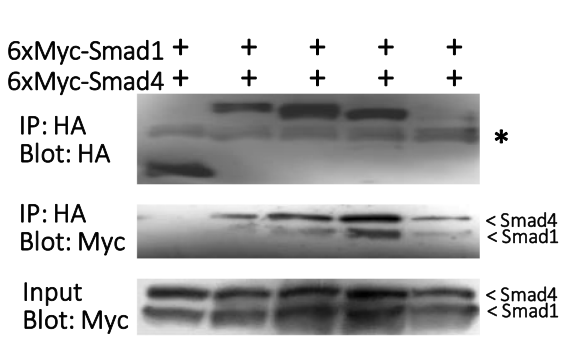

Merge (4848)

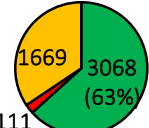

Merge (398)

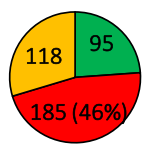

availability of these interactors regulates NPC fate determination. Among SoxC family genes, expression of Sox 11 is abundant in neurogenic (E11) NPCs, but decreases dramatically at the gliogenic stage (E14) (Figs. 4D, 5A). We therefore overexpressed and knocked down Sox11 in E14 and E11 NPCs, respectively, and checked the BMP2 responsiveness of the NPCs. We observed that E14 NPCs regained their neurogenic potential upon Sox11 forced expression; we detected dramatic induction of Neurog1 (Fig. 5B) and Tubb3-positive neurons (Supplemental Fig. $\mathrm{S} 7 \mathrm{~A}$ ) in response to BMP2 stimulation, neither of which
Figure 4. Smads interact with SoxC and SoxE family proteins and co-occupy Smad-BSs. $(A)$ Classification of Smad-BSs identified in this study or reported in mesodermal cells based on chromatin state in NPCs. Chromatin openness was categorized into four states-E11 uniquely open (blue), E14 uniquely open (red), E11/14 commonly open (magenta), or none of these (black) - using ATAC-seq data. (B,left) Venn diagrams showing the number and percentage of Smad co-occupancy in Sox4- or Sox9-bound sites. (Right) Sox4/9 and Smad cobound sites were divided into either E11 unique (green), E14 unique (red), or E11/14 common (orange) Smad-BSs as shown in the pie charts. $(C)$ Myc-tagged Smadl and Smad4 were transfected with HA-tagged Sox family proteins into HEK293T cells together with ALK3-QD and immunoprecipitated using anti-HA antibody. Nonspecific bands observed in the HA blot are indicated by an asterisk. $(D)$ Lysates of BMP2-stimulated E11 or E14 NPCs were precipitated by antiphospho-Smad1/5/9 antibody and blotted with the indicated antibodies.

Table 1. List of transcription factors/cofactors overrepresented around Smad-BSs

\begin{tabular}{|c|c|c|c|c|c|c|c|c|}
\hline \multicolumn{3}{|c|}{ E11 unique Smad-BSs } & \multicolumn{3}{|c|}{ E14 unique Smad-BSs } & \multicolumn{3}{|c|}{ E11/14 common Smad-BSs } \\
\hline Gene & $\log P$-value & Enrichment & Gene & Log $P$-value & Enrichment & Gene & Log $P$-value & Enrichment \\
\hline Smad4 & -324 & 63.4 & Foxp1 & -149.9 & 34.8 & Med1 & -324 & 147.1 \\
\hline Trim28 & -324 & 61.8 & Olig2 & -97.9 & 20.3 & Atrx & -324 & 123.2 \\
\hline Ash2I & -324 & 59.7 & AscI1 & -89.5 & 31.9 & Npas3 & -324 & 114.2 \\
\hline Sox4 & -324 & 57.8 & Kdm $6 \mathrm{~b}$ & -80.5 & 62.3 & Kdmla & -324 & 87.9 \\
\hline Brd4 & -324 & 53.2 & Nfia & -77.8 & 32.0 & Zic1 & -324 & 83.4 \\
\hline Hdac3 & -324 & 51.1 & Stat3 & -73.4 & 219.0 & $\mathrm{Kdm} 6 \mathrm{~b}$ & -324 & 81.8 \\
\hline Gfil & -324 & 50.8 & Tcf3 & -69.3 & 45.0 & Smad3 & -324 & 80.7 \\
\hline Sirt1 & -324 & 49.3 & Atrx & -68.0 & 105.0 & Brd4 & -324 & 80.3 \\
\hline Cbx3 & -324 & 49.0 & Kdmla & -63.7 & 35.7 & Tcf3 & -324 & 79.2 \\
\hline Kdmla & -324 & 47.9 & Sox9 & -48.3 & 153.0 & Ash2I & -324 & 77.8 \\
\hline Zic1 & -324 & 44.5 & Smad3 & -48.1 & 53.7 & Gfil & -324 & 64.2 \\
\hline Ctcf & -324 & 43.8 & Ep300 & -42.4 & 48.3 & Ctcf & -324 & 61.1 \\
\hline Med1 & -324 & 41.5 & Npas3 & -38.2 & 44.3 & Stat3 & -324 & 43.4 \\
\hline AscI1 & -324 & 40.2 & Sox 2 & -32.8 & 56.5 & Foxp1 & -324 & 37.3 \\
\hline Npas3 & -324 & 36.9 & Ctcf & -30.5 & 7.6 & Мус & -324 & 36.8 \\
\hline
\end{tabular}

occurred in control gliogenic (E14) NPCs. Conversely, knockdown of Sox11 in E11 NPCs greatly inhibited Neurog1 induction after BMP2 treatment (Supplemental Fig. $\mathrm{S} 7 \mathrm{~B}$ ), suggesting that the expression level of Sox11 regulates neuronal differentiation induced by BMP2.

In contrast to Sox11, the SoxE gene Sox8 was dramatically up-regulated in E14 NPCs compared with E11 NPCs, and BMP2 treatment further induced its expression (Fig. 5A; Supplemental Fig. S2C). We therefore overexpressed Sox8 in neurogenic (E11) NPCs and differentiated them with BMP2. However, we could not detect any 
induction of later-stage-specific Smad target genes (Fig. 5C), even though Nfia overexpression had that effect (Fig. 3E). As we have recently reported (Takouda et al. 2021) Sox8 cannot induce DNA demethylation of the Gfap promoter, suggesting that Sox8 cannot make NPCs override epigenetic barriers. However, when a Gfap promoter-fused luciferase construct was introduced into NPCs, we did see a slight enhancement of Gfap promoter activation by Sox 8 in the presence of BMP2 (Fig. 5D), suggesting that DNA methylation is what precludes Sox8Smad binding to the Gfap promoter. Interestingly, we observed that overexpression of Sox11, the neurogenic partner of Smads, dramatically reduces BMP2-induced Gfap promoter activation even in Sox8-overexpressing NPCs (Fig. 5D). Altogether, differences in the availability of distinct Sox family proteins as Smad interactors are implicated in the regulation of the response to BMP2, controlling property switching of NPCs during development.

\section{Discussion}

We have demonstrated in this study that the chromatin accessibility of Smad-BSs dramatically changes genomewide during cortical development, and, as a consequence, that neuronal differentiation in mid-gestational NPCs, but astrocytic differentiation in late-gestational NPCs, is induced in response to the differentiation cue from BMP2. As a representative gene, we focused intensively on the regulation of Neurog1, which plays a central role in neuronal differentiation in the brain (Fode et al. 2000), and showed that its responsiveness to BMP2 clearly differs between E11 and E14 NPCs (Supplemental Fig. S2C). Neurog1 outcompetes STAT3 for binding to the p300/ CBP-Smad1 complex, an essential complex for astrocytic differentiation, leading to suppression of NPC differentia- tion into astrocytes (Sun et al. 2001). Although transcriptional regulation, including epigenetic mechanisms, for Neurog1 is well characterized (Hirabayashi et al. 2009; Onoguchi et al. 2012; Tsuboi et al. 2018), our global analyses of Smad-BSs have uncovered an additional and key layer of Neurog1 regulatory complexity to explain the temporally controlled property alteration of NPCs.

It is now widely accepted that the Smad complex (composed of R-Smads and common Smad4) itself has relatively weak affinity for DNA, and limited specificity; therefore, its interaction with other sequence-specific transcription factors that either actively recruit the Smad complex or stabilize its DNA binding is important (Morikawa et al. 2013; Gaarenstroom and Hill 2014). We have identified Sox11 and Sox8 as developmental stagedependent Smad-interacting partners. Since the expression of Sox11 and Sox8 is high in neurogenic and astrocytic NPCs, respectively, their interaction with Smads was observed at different developmental stages (Fig. 4D). If opposed lineage-specific Smad partners are expressed in the same developmental period, they seem to compete for Smad binding, as we observed that Nfia and Sox8 overexpression in neurogenic NPCs reduced Neurog1 induction after BMP2 treatment (Figs. 3E, 5C). Such an effect was clearly observed in the reporter assay, where simultaneous expression of Sox8 and Sox11, in contrast to Sox 8 single expression, reduced Gfap expression (Fig. 5D).

We identified lineage-specific Smad interactors, and their complexes seem to regulate target gene expression in a context-dependent manner. As noted earlier, subsets of Smad target genes behave oppositely in E11 and E14 NPCs (Supplemental Fig. S3D). We looked in detail at the Hes5, Hes6, Olig1, and Olig2 loci, but the Smad-binding patterns and ATAC-seq signatures around these loci were similar at the two developmental stages (Supplemental Fig. S8). Nevertheless, BMP2 enhanced gene
A

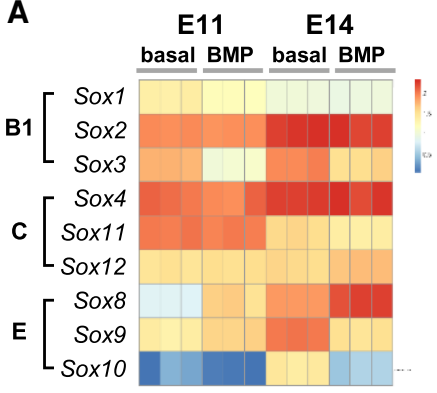

B

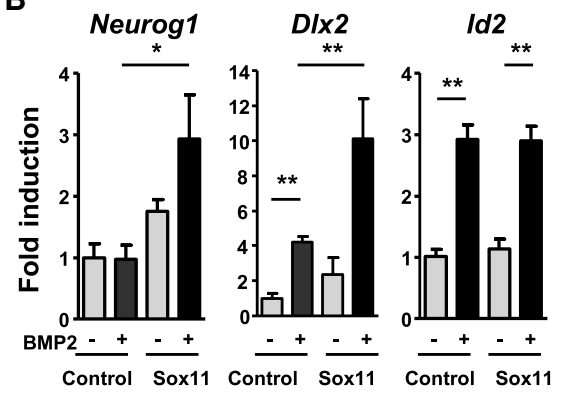

Figure 5. Smad-interacting partners (Sox 11 for neurogenic and Sox8 for astrocytic differentiation) ensure Smad-regulated fate-specific gene induction. (A) Expression levels of SoxB1/C/E family genes in E11 or E14 NPCs are depicted as a heat map. (B) E14-derived NPCs were infected with lentivirus engineered to express Sox11 and cultured for $2 \mathrm{~d}$ with bFGF, and mRNA was isolated before and $24 \mathrm{~h}$ after BMP2 stimulation. Real-time RT-PCR data are show as means \pm $\mathrm{SD}(n=3) .\left(^{* *}\right) P<0.01,\left(^{*}\right) P<0.05$ by one-way ANOVA with Bonferroni's multiple comparison test. $(C)$ E11derived NPCs were infected with lentivirus to express Sox8 (red) or control (black) and cultured for $2 \mathrm{~d}$ with bFGF, and mRNA was isolated before and $24 \mathrm{~h}$ after BMP2 treatment. Real-time RT-PCR data are shown as means $\pm \mathrm{SD}(n=3)$. $(D)$ Luciferase assay of Gfap promoter in E14-derived NPCs. Together with the reporter, Sox8 or Sox11 were cotransfected as indicated, and the cells were stimulated with BMP2 for $24 \mathrm{~h}$ (gray). Relative light units compared with control without BMP2 (white) are indicated. Bar graph shows means $\pm \operatorname{SD}(n=4)$. (**) $P<0.01,(*) P<0.05$ by one-way ANOVA with Bonferroni's multiple comparison test. 
expression at one stage, but repressed it at the other, suggesting that Smad contexts in each CRE differ in neurogenic and gliogenic NPCs. Smads are known to form complexes with coactivators such as p300/CBP histone acetyltransferases, or with corepressors associated with histone deacetylases, to regulate gene expression (Miyazono et al. 2007); therefore, Smad interaction with an activator or a repressor complex defines the direction of gene induction. Hes family members repress neurogenesis (Kageyama et al. 2007), and we found that BMP2 repressed Hes 5 and Hes6 expression in neurogenic NPCs but induced their expression in gliogenic NPCs (Supplemental Fig. S3D). Similarly, regarding Olig family gene function for oligodendrocyte specification, BMP2 repressed Olig1 and Olig2 expression to ensure astrocytic differentiation in later-stage NPCs (Supplemental Fig. S3D), emphasizing the highly organized nature of the gene induction system downstream from BMP signaling toward the neuronal-toastrocytic differentiation switch.

We also showed that Nfia, whose expression becomes higher in later-stage NPCs, confers gliogenic competence on NPCs by inducing DNA demethylation around SmadBSs of astrocytic genes (Fig. 3D,E). Since Smads preferentially bind to cognate sites within open chromatin regions, epigenetic regulation also plays a crucial role to control NPC fate determination. This is clear in our result that forced expression of the later-stage-specific Smad partner, Sox8, is incapable of generating astrocytes in neurogenic NPCs even upon BMP2 stimulation (Fig. 5C). While further studies will be necessary to clarify the involvement of histone modifications in this regulation, we have nevertheless identified Trim28, Hdac3, and Sirt1 as factors that are overrepresented around E11 unique Smad-BSs (Table 1). Because these three factors are known to control histone methylation and acetylation, and thus to repress gene expression (Prozorovski et al. 2008; Czerwińska et al. 2017; Li et al. 2019), it is conceivable that they act to modulate $\mathrm{H} 3 \mathrm{~K} 27 \mathrm{me} 3$, because we observed an increase of methylation level around these E11 unique Smad-BSs (Fig. 3A). Conversely, a decrease in H3K27me3 around E14 unique Smad-BSs was observed (Fig. 3A), and this may be governed by the histone H3K27-specific demethylase Jumonji D3 (Jmjd3). Interaction of Jmjd3 with Smad2/3, transcription factors downstream from TGF- $\beta$ signaling, has been reported (Dahle et al. 2010; Kim et al. 2011), and, furthermore, genomewide analyses of Jmjd3 and Smad3 binding sites in NPCs revealed that these two factors co-occupied the same regions to activate the neural developmental program (Estarás et al. 2012). In contrast to TGF- $\beta$ signaling, the involvement of Jmjd3 in BMP signaling has only once been reported (Akizu et al. 2010); however, we detected a Smad-Jmjd3 interaction (Supplemental Fig. S9A), and $50 \%$ of the Smad3 binding sites identified in the above study overlapped with our Smad-BSs (Supplemental Fig. S9B), suggesting a Smads and Jmjd3 complex as the candidate machinery to induce demethylation of $\mathrm{H} 3 \mathrm{~K} 27 \mathrm{me} 3$ around E14 unique Smad-BSs.

We demonstrate here for the first time that SoxC and SoxE family proteins interact with BMP signal transducer
Smads, and that these partners regulate target gene expression to control NPC differentiation. We have shown that the switching of Smad partner from Sox 11 to Sox8 induces the change of neurogenic to astrocytic gene induction, but we do not exclude the possibility that some other lineage-specific Smad interactors exist, since about half of the Sox4 binding sites overlapped with our SmadBSs, whereas only $10 \%$ overlapped for Sox9 (Fig. 4B). The association of Olig1 and Nfia with Smads has been reported (Luciakova et al. 2008; Motizuki et al. 2013), and these gliogenic transcription factors may therefore also recruit Smads to additional target loci in NPCs. We observed fewer Smad-BSs in gliogenic E14 NPCs (5213) than in neurogenic E11 NPCs $(13,538)$ (Fig. 1D). Although warranting further study, this smaller number of SmadBSs and lower enrichment of Smad signal in E14 NPCs (Supplemental Fig. S3E) may be atrributable to the number of existing Smad interactors at each stage. As noted above, sequence-specific transcription factors are necessary for the stabilization of Smads-DNA binding. Therefore, if many distinct interactors exist at the same developmental stages, they are likely to compete with each other for Smad binding, as we observed for Sox8 and Sox 11 (Fig. 5D). Such competition may reduce the amount of Smads on respective Smad-BSs, since Smads are dispersed across many genomic loci containing binding sites of individual Smads' interactors simultaneously, reflecting a lower signal for Smad binding around E14 Smad-BSs compared with that obtained for E11.

Figure 6 summarizes our results to illustrate the exquisite regulation of the NPC property change through altered accessibility of chromatin to Smads. We found that epigenetic modifications around Smad-BSs dramatically change throughout the genome during cortical development, conferring the neuronal property in mid-gestational NPCs, but astrocytic fate in late-gestational NPCs. Since Smads function as transducers for endogenous ligands in the

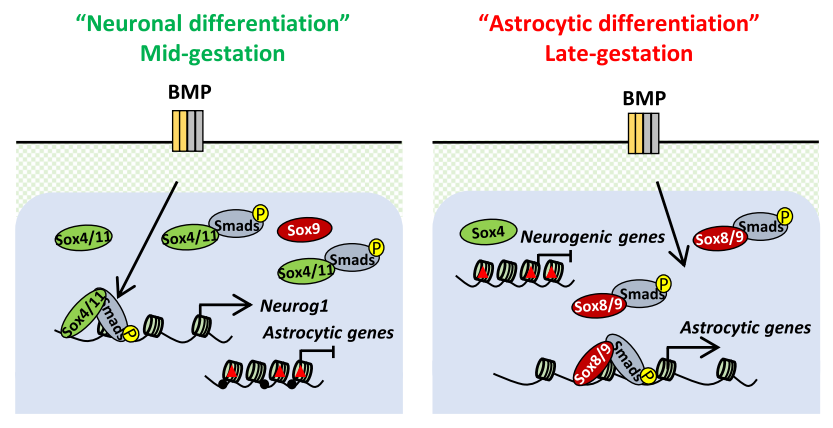

Figure 6. Schematic model for the effects of BMPs on developmentally different NPCs. In mid-gestation, chromatin accessibility of astrocytic genes is restricted by epigenetic modifications such as DNA methylation and H3K27 trimethylation; however, during NPC progression, these repressive epigenetic marks are removed and, in turn, neurogenic genes acquire $\mathrm{H} 3 \mathrm{~K} 27$ trimethylation, leading to a less accessible chromatin state. Upon BMP stimulation, activated Smads bind to reachable consensus sequences, but developmental stage-specific Smad partners further ensure Smad-regulated fate-specific gene induction. 
neocortex (Hegarty et al. 2013), NPCs delicately control their status via the interplay between cell-extrinsic cues and -intrinsic epigenetic programs to generate the appropriate numbers of each neural cell type. Lineage-specific interactors of Smads further orchestrate NPC fate choice to establish proper brain development. A key question to address next is how the expression levels of multiple lineage-specific transcription factors are regulated in NPCs. As we showed in Figure 1B, the expression of well-characterized neuronal transcription factors such as Dlx1/2, Emx1, Msx1/2, and Neurog1 is high in E11 NPCs but dramatically down-regulated in gliogenic NPCs. Conversely, gliogenic genes like Gfap, Nfia/x, and Sox8 are predominantly induced in later-stage NPCs, indicating that the basal expression level of fate-regulating transcription factors is already systematized in each individual cell. Sonic Hedgehog (Shh) is well known to induce Olig1/2 expression (Lu et al. 2000), and Notch-induced Nfia can activate Sox8 and Olig1/2 (Takouda et al. 2021). A recent study in mouse cerebellum demonstrated that the temporal dynamics of the BMP/Smad signaling gradient in NPCs orchestrates the transition from the early to the late phase of neurogenesis during development ( $\mathrm{Ma}$ et al. 2020). Therefore, together with Notch and Shh, we speculate that the Smad signal itself promotes NPCs' developmental progression. Because activation of the Smad signal is observed from early developmental stages in NPCs (Falk et al. 2008) and BMP2 induces expression of some gliogenic transcription factors in neurogenic NPCs (Supplemental Fig. S2C), the BMP-Smad signal may function to induce both progression and differentiation of NPCs. As has been shown, our current study sheds further light on NPC fate switching in the transition of the two distinct developmental phases; however, other questions, such as how these epigenetic landscapes are established and how they are altered during these phases, remain as the next challenging issues to be resolved.

\section{Materials and methods}

\section{Mouse NPCs}

Timed pregnant mice were used to prepare NPCs as described previously (Namihira et al. 2009). C57BL/6NCrSlc (Japan SLC) were used for the next-generation sequencing (NGS)-based studies; otherwise, ICR/SLC (Japan SLC) were used because of the abundance of embryos. In brief, telencephalons from E11 or E14 mice were triturated in Hanks' balanced salt solution by gentle pipetting. After centrifugation, the cell pellet was suspended in N2-supplemented DMEM/F12 containing $10 \mathrm{ng} / \mathrm{mL}$ basic fibroblast growth factor (PeproTech) and cultured on dishes coated with poly-L-ornithine and fibronectin. Cells were cultured for 2 $\mathrm{d}$ for E11 and $5 \mathrm{~d}$ for E14 to enrich the NPC population.

\section{$R N A$ isolation and $c D N A$ preparation}

Total RNAs were extracted with Sepasol-RNA I Super G (Nacalai Tesque) from E11 or E14 NPCs before and $24 \mathrm{~h}$ after BMP2 stimulation $(50 \mathrm{ng} / \mathrm{mL})$. Reverse transcription was performed from 500 ng of total RNA using a SuperScript VILO cDNA synthesis kit (Thermo Fisher Scientific). For cDNA library preparation,
mRNAs were isolated from total RNA with an NEBNext poly (A) mRNA magnetic isolation module (New England Biolabs), and the purified mRNAs were subjected to cDNA library construction using an NEBNext Ultra Directional RNA library preparation kit for Illumina (New England Biolabs) following the manufacturer's protocols.

RNA-seq and bioinformatics

Libraries were evaluated and quantified using an Agilent 2100 Bioanalyzer with high-sensitivity DNA chip (Agilent Technologies) and sequenced with an Illumina HiSeq 3000, and 100-bp paired-end reads were generated by the Platform for Advanced Genome Science (PAGS). The sequencing data were submitted to the DDBJ under accession number DRA007214. Obtained reads were assessed with FastQC, and mapped to the mouse mm10 genome using TopHat/Bowtie2. To determine BMP2-responsive genes, generated BAM files were accessed with Cuffdiff, and $q$-values $<0.05$ were considered significant. A heat map was created using iDEP (http://bioinformatics.sdstate.edu) with the top 2500 variable genes, and $k=6$ for the K-means clustering. GSEA was carried out using signal to noise as the ranking metric and with the "weighted" scoring scheme. Together with the registered GO terms, which were downloaded from Mouse Genome Informatics, self-assigned gene sets were created using public RNA-seq data (Zhang et al. 2014) and analyzed. Neuron- and astrocyte-enriched gene sets included 1055 and 720 genes with $\log _{2}$ $\mathrm{FC}>1$ and $\mathrm{FPKM}>1$, respectively.

\section{ChIP-seq and data analyses}

To identify Smad-binding sites (Smad-BSs), ChIP samples were prepared from E11 or E14 NPCs before and $1 \mathrm{~h}$ after BMP2 stimulation $(50 \mathrm{ng} / \mathrm{mL}$ ) using anti-phospho-Smad1/5/9 antibody (Cell Signaling Technology 13820). After conventional ChIP, sequencing libraries were made according to instructions in the NEBNext Ultra II DNA library preparation kit for Illumina (New England Biolabs) and sequenced with an Illumina HiSeq 3000, and 36-bp single-end reads were generated by PAGS. The sequencing data were submitted to the DDBJ under accession number DRA007215. Obtained reads were assessed with FastQC, and mapped to the mouse mm10 genome using Bowtie2. Peak calling for Smad-BSs was performed with MACS2 using a BMP2 untreated sample as control. To investigate the biological meaning of Smad-BSs in E11 and E14 NPCs, GREAT (v3; http://great .stanford.edu) was used. To perform de novo motif discovery, 51-bp sequences surrounding the summits of Smad-BSs were analyzed using DREME (v5.3.3) in the MEME suite (http $: / /$ meme-suite.org). To search for transcription factors that bind to Smad-BSs, the public ChIP-seq database ChIP-Atlas (https ://chip-atlas.org) was used with the cell type "neural." To evaluate histone modifications in neurogenic and gliogenic NPCs, ChIP samples were prepared from untreated E11 or E14 NPCs using anti-H3K27me3 (MBL MAB10323) antibodies. After ChIP and library preparation, libraries were sequenced with an Illumina HiSeq 2500, and 50-bp single-end reads were generated. The sequencing data were submitted to the GEO under accession number GSE174306. Publicly available ChIP-seq data in the GEO (GSE36673, GSE85797, GSE117997, and GSE120894) were used to develop our studies.

\section{ATAC-seq}

ATAC sequencing was carried out in duplicate as described previously (Harada et al. 2018). In brief, naked DNA from untreated 
E11 or E14 NPCs $(50,000$ cells) was incubated with the Tn5 adaptor DNA complex for $30 \mathrm{~min}$ at $37^{\circ} \mathrm{C}$, and DNA fragments in the reaction mixture were purified using a Qiagen minielute kit. After purification, the fragments were amplified using custom Nextera PCR primers for 15 cycles, and the library fragments were purified using a Qiagen minielute kit. Libraries were size-selected with AMPure XP beads, and paired-end sequences of $100 \mathrm{bp}$ were generated by PAGS on the Illumina HiSeq 3000 platform. The sequencing data were submitted to the DDBJ under accession number DRA007213. ATAC-seq captions were obtained from the GenomeJack genome browser.

\section{Immunocytochemistry}

Immunocytochemistry was performed in NPCs as previously described (Nakashima et al. 1999; Honda et al. 2017).

\section{Lentivirus preparation}

The lentiviral vector pLEMPRA was used to overexpress Nfia, Sox8, and Sox11. For knockdown experiments, pLLX vector was used. HEK293T cells were transfected with pLEMPRA or pLLX together with the lentivirus constructs VSVG, Rev, and MDL using polyethylenimine (Polysciences). The next day, the medium was replaced with N2-supplemented DMEM/F12 and the cells were cultured for $48 \mathrm{~h}$. The supernatant was centrifuged at $4^{\circ} \mathrm{C}$ to collect virus.

\section{$R T-q P C R$}

Total RNAs were extracted with Sepasol-RNA I Super G (Nacalai Tesque), and 500-ng aliquots of total RNAs were subjected to reverse transcription using a SuperScript VILO cDNA synthesis kit (Thermo Fisher Scientific). Quantitative real-time PCR (qPCR) was performed with the Stratagene Mx300p (Agilent Technologies) using a KAPA SYBR Fast qPCR kit (Nippon Genetics). Expression levels of each gene were normalized to Actb and calculated relative to the control.

\section{Western blotting}

To perform coimmunoprecipitation, pcDNA3-6xMyc-Smad1, pcDNA3-6xMyc-Smad4, and pLEMPRA-HA-(Sox $2 / 4 / 8 / 9 / 11)$ were transfected into HEK293T cells using polyethylenimine. To activate BMP signals, a constitutively active mutant of ALK3 (ALK3-QD) was cotransfected. Two days after transfection, the cells were lysed in lysis buffer, and HA-tagged proteins were immunoprecipitated with $1 \mu \mathrm{g}$ of anti-HA antibody (MBL M180-3) and $30 \mu \mathrm{L}$ of magnetic Dynabeads M-280 sheep antimouse IgG. Lysate inputs and immunoprecipitated samples were immunoblotted with anti-HA and anti-Myc antibody (MBL M047-3). For coimmunoprecipitation in NPCs, anti-phospho-Smad1/5/9 antibody (Cell Signaling Technology 13820) was used to immunoprecipitate, and blotting was performed with anti-Sox8 (Abcam ab104245) or anti-Sox11 (Santa Cruz Biotechnology sc-20096) antibodies.

\section{Luciferase assay}

The luciferase assay was performed in E14-derived NPCs as previously described (Nakashima et al. 1999; Honda et al. 2017).

\section{Competing interest statement}

The authors declare no competing interests.

\section{Acknowledgments}

We thank M.E. Greenberg for pLLX vector, H. Kimura for antiH3K27me3 antibodies, and I. Smith for proofreading the manuscript. We appreciate technical assistance from the Research Support Center, Kyushu University Graduate School of Medical Sciences. We thank N. Yamamoto and M. Otsuka for technical advice for NGS. This work was supported by The Ministry of Education, Culture, Sports, Science, and Technology/Japan Society for the Promotion of Science KAKENHI (JP16H06527 and JP16K21734 to K.N., and JP26710003 and JP20K06875 to S.K.), Japan Science and Technology Agency CREST (JPMJCR16G1 to Y.O. and H.K.), the Naito Foundation (S.K.), and the Platform for Advanced Genome Science (PAGS; JP17H05647 to S.K.).

Author contributions: K.N. and S.K. conceived the study. S.K., J.T., T.N., and M.H. acquired the data. S.K. analyzed and interpreted the data and prepared the manuscript. T.I. supervised the informatics. K.I., Y.O., S.S., and H.K. acquired the resources. K.N. critically revised the manuscript. S.K. and K.N. acquired the funding.

\section{References}

Akizu N, Estarás C, Guerrero L, Martí E, Martínez-Balbás MA. 2010. H3k27me3 regulates BMP activity in developing spinal cord. Development 137: 2915-2925. doi:10.1242/dev.049395

Albert M, Huttner WB. 2018. Epigenetic and transcriptional prepatterning - an emerging theme in cortical neurogenesis. Front Neurosci 12: 359. doi:10.3389/fnins.2018.00359

Baik J, Magli A, Tahara N, Swanson SA, Koyano-Nakagawa N, Borges L, Stewart R, Garry DI, Kawakami Y, Thomson JA, et al. 2016. Endoglin integrates BMP and Wnt signalling to induce haematopoiesis through JDP2. Nat Commun 7: 13101. doi:10.1038/ncomms13101

Barnabé-Heider F, Wasylnka JA, Fernandes KJL, Porsche C, Sendtner M, Kaplan DR, Miller FD. 2005. Evidence that embryonic neurons regulate the onset of cortical gliogenesis via cardiotrophin-1. Neuron 48: 253-265. doi:10.1016/j.neuron.2005 .08 .037

Bonaguidi MA, McGuire T, Hu M, Kan L, Samanta J, Kessler JA. 2005. LIF and BMP signaling generate separate and discrete types of GFAP-expressing cells. Development 132: 5503 5514. doi:10.1242/dev.02166

Braccioli L, Vervoort SJ, Puma G, Nijboer CH, Coffer PJ. 2018. SOX4 inhibits oligodendrocyte differentiation of embryonic neural stem cells in vitro by inducing Hes5 expression. Stem Cell Res 33: 110-119. doi:10.1016/j.scr.2018.10.005

Burmistrova O, Olias-Arjona A, Lapresa R, Jimenez-Blasco D, Eremeeva T, Shishov D, Romanov S, Zakurdaeva K, Almeida A, Fedichev PO, et al. 2019. Targeting PFKFB3 alleviates cerebral ischemia-reperfusion injury in mice. Sci Rep 9: 11670. doi:10 .1038/s41598-019-48196-z

Cole AE, Murray SS, Xiao J. 2016. Bone morphogenetic protein 4 signalling in neural stem and progenitor cells during development and after injury. Stem Cells Int 2016: 9260592. doi:10 $.1155 / 2016 / 9260592$

Czerwińska P, Mazurek S, Wiznerowicz M. 2017. The complexity of TRIM28 contribution to cancer. I Biomed Sci 24: 63. doi:10.1186/s12929-017-0374-4 
Dahle Ø, Kumar A, Kuehn MR. 2010. Nodal signaling recruits the histone demethylase Jmjd3 to counteract polycomb-mediated repression at target genes. Sci Signal 3: ra48. doi:10.1126/sci signal.2000841

Duong TAD, Hoshiba Y, Saito K, Kawasaki K, Ichikawa Y, Matsumoto N, Shinmyo Y, Kawasaki H. 2019. FGF signaling directs the cell fate switch from neurons to astrocytes in the developing mouse cerebral cortex. J Neurosci 39: 6081-6094. doi:10 .1523/JNEUROSCI.2195-18.2019 https://www.jneurosci.org/ content/39/31/6081.abstract

Eiraku M, Watanabe K, Matsuo-Takasaki M, Kawada M, Yonemura S, Matsumura M, Wataya T, Nishiyama A, Muguruma K, Sasai Y. 2008. Self-organized formation of polarized cortical tissues from ESCs and its active manipulation by extrinsic signals. Cell Stem Cell 3: 519-532. doi:10.1016/j.stem.2008.09 .002

Estarás C, Akizu N, García A, Beltrán S, de la Cruz X, MartínezBalbás MA. 2012. Genome-wide analysis reveals that $\mathrm{Smad} 3$ and JMJD3 HDM co-activate the neural developmental program. Development 139: 2681-2691. doi:10.1242/dev.078345

Falk S, Wurdak H, Ittner LM, Ille F, Sumara G, Schmid M-T, Draganova K, Lang KS, Paratore C, Leveen P, et al. 2008. Brain area-specific effect of TGF- $\beta$ signaling on Wnt-dependent neural stem cell expansion. Cell Stem Cell 2: 472-483. doi:10 $.1016 /$ j.stem.2008.03.006

Fode C, Ma Q, Casarosa S, Ang SL, Anderson DJ, Guillemot F. 2000. A role for neural determination genes in specifying the dorsoventral identity of telencephalic neurons. Genes Dev 14: $67-80$.

Gaarenstroom T, Hill CS. 2014. TGF- $\beta$ signaling to chromatin: how Smads regulate transcription during self-renewal and differentiation. Semin Cell Dev Biol 32: 107-118. doi:10.1016/j .semcdb.2014.01.009

Gaspard N, Bouschet T, Hourez R, Dimidschstein J, Naeije G, van den Ameele I, Espuny-Camacho I, Herpoel A, Passante L, Schiffmann SN, et al. 2008. An intrinsic mechanism of corticogenesis from embryonic stem cells. Nature 455: 351-357. doi:10.1038/nature07287

Harada A, Maehara K, Ono Y, Taguchi H, Yoshioka K, Kitajima Y, Xie Y, Sato Y, Iwasaki T, Nogami J, et al. 2018. Histone H3.3 sub-variant $\mathrm{H} 3 \mathrm{~mm} 7$ is required for normal skeletal muscle regeneration. Nat Commun 9: 1400. doi:10.1038/s41467-01803845-1

Hegarty SV, O'Keeffe GW, Sullivan AM. 2013. BMP-Smad 1/5/8 signalling in the development of the nervous system. Prog Neurobiol 109: 28-41. doi:10.1016/j.pneurobio.2013.07.002

Hirabayashi Y, Suzki N, Tsuboi M, Endo TA, Toyoda T, Shinga J, Koseki H, Vidal M, Gotoh Y. 2009. Polycomb limits the neurogenic competence of neural precursor cells to promote astrogenic fate transition. Neuron 63: 600-613. doi:10.1016/j .neuron.2009.08.021

Honda M, Nakashima K, Katada S. 2017. PRMT1 regulates astrocytic differentiation of embryonic neural stem/precursor cells. J Neurochem 142: 901-907. doi:10.1111/jnc.14123

Kageyama R, Ohtsuka T, Kobayashi T. 2007. The Hes gene family: repressors and oscillators that orchestrate embryogenesis. Development 134: 1243-1251. doi:10.1242/dev.000786

Kawamura Y, Katada S, Noguchi H, Yamamoto H, Sanosaka T, Iihara K, Nakashima K. 2017. Synergistic induction of astrocytic differentiation by factors secreted from meninges in the mouse developing brain. FEBS Lett 591: 3709-3720. doi:10.1002/1873-3468.12881

Kim SW, Yoon S-J, Chuong E, Oyolu C, Wills AE, Gupta R, Baker J. 2011. Chromatin and transcriptional signatures for Nodal signaling during endoderm formation in hESCs. Dev Biol 357: 492-504. doi:10.1016/j.ydbio.2011.06.009

Klemm SL, Shipony Z, Greenleaf WJ. 2019. Chromatin accessibility and the regulatory epigenome. Nat Rev Genet 20: 207-220. doi:10.1038/s41576-018-0089-8

Klum S, Zaouter C, Alekseenko Z, Björklund ÅK, Hagey DW, Ericson J, Muhr J, Bergsland M. 2018. Sequentially acting SOX proteins orchestrate astrocyte- and oligodendrocyte-specific gene expression. EMBO Rep 19: e46635. doi:10.15252/ embr.201846635

Kohyama J, Sanosaka T, Tokunaga A, Takatsuka E, Tsujimura K, Okano H, Nakashima K. 2010. BMP-induced REST regulates the establishment and maintenance of astrocytic identity. I Cell Biol 189: 159-170. doi:10.1083/jcb.200908048

Kuhlbrodt K, Herbarth B, Sock E, Hermans-Borgmeyer I, Wegner M. 1998. Sox10, a novel transcriptional modulator in glial cells. I Neurosci 18: 237-250. doi:10.1523/JNEUROSCI.1801-00237.1998

Li W, Cogswell CA, LoTurco JJ. 1998. Neuronal differentiation of precursors in the neocortical ventricular zone is triggered by BMP. I Neurosci 18: 8853-8862. doi:10.1523/JNEUROSCI .18-21-08853.1998

Li L, Jin J, Yang X-J. 2019. Histone deacetylase 3 governs perinatal cerebral development via neural stem and progenitor cells. iScience 20: 148-167. doi:10.1016/j.isci.2019.09.015

Lu QR, Yuk D, Alberta JA, Zhu Z, Pawlitzky I, Chan J, McMahon AP, Stiles CD, Rowitch DH. 2000. Sonic hedgehog-regulated oligodendrocyte lineage genes encoding bHLH proteins in the mammalian central nervous system. Neuron 25: 317329. doi:10.1016/S0896-6273(00)80897-1

Luciakova K, Kollarovic G, Barath P, Nelson BD. 2008. Growthdependent repression of human adenine nucleotide translocator-2 (ANT2) transcription: evidence for the participation of smad and Sp family proteins in the NF1-dependent repressor complex. Biochem J 412: 123-130. doi:10.1042/BJ20071440

Ma TC, Vong KI, Kwan KM. 2020. Spatiotemporal decline of BMP signaling activity in neural progenitors mediates fate transition and safeguards neurogenesis. Cell Rep 30: 36163624.e4. doi:10.1016/j.celrep.2020.02.089

Miller FD, Gauthier AS. 2007. Timing is everything: making neurons versus glia in the developing cortex. Neuron 54: 357-369. doi:10.1016/j.neuron.2007.04.019

Miyazono K, Maeda S, Imamura T. 2007. Smad transcriptional co-activators and co-repressors. In Proteins and cell regulation (ed. Dijke P, Heldin CH), pp. 277-293. Springer, Dordrecht, Netherlands.

Morikawa M, Koinuma D, Tsutsumi S, Vasilaki E, Kanki Y, Heldin C-H, Aburatani H, Miyazono K. 2011. ChIP-seq reveals cell type-specific binding patterns of BMP-specific smads and a novel binding motif. Nucleic Acids Res 39: 87128727. doi:10.1093/nar/gkr572

Morikawa M, Koinuma D, Miyazono K, Heldin C-H. 2013. Genome-wide mechanisms of smad binding. Oncogene 32: 1609-1615. doi:10.1038/onc.2012.191

Motizuki M, Isogaya K, Miyake K, Ikushima H, Kubota T, Miyazono K, Saitoh M, Miyazawa K. 2013. Oligodendrocyte transcription factor 1 (Olig1) is a smad cofactor involved in cell motility induced by transforming growth factor- $\beta$. I Biol Chem 288: 18911-18922. doi:10.1074/jbc.M113.480996

Nakagawa T, Wada Y, Katada S, Kishi Y. 2020. Epigenetic regulation for acquiring glial identity by neural stem cells during cortical development. Glia 68: 1554-1567. doi:10.1002/glia .23818

Nakashima K, Yanagisawa M, Arakawa H, Kimura N, Hisatsune T, Kawabata M, Miyazono K, Taga T. 1999. Synergistic 
signaling in fetal brain by STAT3-Smad1 complex bridged by p300. Science 284: 479-482. doi:10.1126/science.284.5413 .479

Nakashima K, Takizawa T, Ochiai W, Yanagisawa M, Hisatsune T, Nakafuku M, Miyazono K, Kishimoto T, Kageyama R, Taga T. 2001. BMP2-mediated alteration in the developmental pathway of fetal mouse brain cells from neurogenesis to astrocytogenesis. Proc Natl Acad Sci 98: 5868-5873. doi:10.1073/ pnas.101109698

Namihira M, Kohyama J, Semi K, Sanosaka T, Deneen B, Taga T, Nakashima K. 2009. Committed neuronal precursors confer astrocytic potential on residual neural precursor cells. Dev Cell 16: 245-255. doi:10.1016/j.devcel.2008.12.014

Onoguchi M, Hirabayashi Y, Koseki H, Gotoh Y. 2012. A noncoding RNA regulates the neurogenin 1 gene locus during mouse neocortical development. Proc Natl Acad Sci 109: 1693916944. doi:10.1073/pnas.1202956109

Paridaen JTML, Huttner WB. 2014. Neurogenesis during development of the vertebrate central nervous system. EMBO Rep 15: 351-364. doi:10.1002/embr.201438447

Potzner MR, Tsarovina K, Binder E, Penzo-Méndez A, Lefebvre V, Rohrer H, Wegner M, Sock E. 2010. Sequential requirement of Sox4 and Sox11 during development of the sympathetic nervous system. Development 137: 775-784. doi:10.1242/dev .042101

Prozorovski T, Schulze-Topphoff U, Glumm R, Baumgart J, Schröter F, Ninnemann O, Siegert E, Bendix I, Brüstle O, Nitsch R, et al. 2008. Sirt1 contributes critically to the redox-dependent fate of neural progenitors. Nat Cell Biol 10: 385-394. doi:10.1038/ncb1700

Ramachandran A, Vizán P, Das D, Chakravarty P, Vogt J, Rogers KW, Müller P, Hinck AP, Sapkota GP, Hill CS. 2018. TGF- $\beta$ uses a novel mode of receptor activation to phosphorylate SMAD1/5 and induce epithelial-to-mesenchymal transition. Elife 7: e31756. doi:10.7554/elife.31756

Renner M, Lancaster MA, Bian S, Choi H, Ku T, Peer A, Chung K, Knoblich JA. 2017. Self-organized developmental patterning and differentiation in cerebral organoids. EMBO $J$ 36: 13161329. doi:10.15252/embj.201694700

Rowitch DH, Kriegstein AR. 2010. Developmental genetics of vertebrate glial-cell specification. Nature 468: 214-222. doi:10.1038/nature09611

Sanosaka T, Imamura T, Hamazaki N, Chai M, Igarashi K, IdetaOtsuka M, Miura F, Ito T, Fujii N, Ikeo K, et al. 2017. DNA methylome analysis identifies transcription factor-based epigenomic signatures of multilineage competence in neural stem/progenitor cells. Cell Rep 20: 2992-3003. doi:10.1016/j .celrep.2017.08.086
Shen Q, Wang Y, Dimos JT, Fasano CA, Phoenix TN, Lemischka IR, Ivanova NB, Stifani S, Morrisey EE, Temple S. 2006. The timing of cortical neurogenesis is encoded within lineages of individual progenitor cells. Nat Neurosci 9: 743-751. doi:10 $.1038 / \mathrm{nn} 1694$

Shu T, Butz KG, Plachez C, Gronostajski RM, Richards LJ. 2003. Abnormal development of forebrain midline glia and commissural projections in Nfia knock-out mice. J Neurosci 23: 203212. doi:10.1523/JNEUROSCI.23-01-00203.2003

Stolt CC, Lommes P, Sock E, Chaboissier M-C, Schedl A, Wegner M. 2003. The Sox 9 transcription factor determines glial fate choice in the developing spinal cord. Genes Dev 17: 16771689. doi:10.1101/gad.259003

Sun Y, Nadal-Vicens M, Misono S, Lin MZ, Zubiaga A, Hua X, Fan G, Greenberg ME. 2001. Neurogenin promotes neurogenesis and inhibits glial differentiation by independent mechanisms. Cell 104: 365-376. doi:10.1016/S0092-8674(01) 00224-0

Takizawa T, Nakashima K, Namihira M, Ochiai W, Uemura A, Yanagisawa M, Fujita N, Nakao M, Taga T. 2001. DNA methylation is a critical cell-intrinsic determinant of astrocyte differentiation in the fetal brain. Dev Cell 1: 749-758. doi:10 .1016/s1534-5807/01)00101-0

Takouda J, Katada S, Imamura T, Sanosaka T, Nakashima K. 2021. SoxE group transcription factor Sox 8 promotes astrocytic differentiation of neural stem/precursor cells downstream of Nfia. Pharmacol Res Perspect 9: e00749. doi:10.1002/ prep2.749

Temple S. 2001. The development of neural stem cells. Nature 414: 112-117. doi:10.1038/35102174

Tsuboi M, Kishi Y, Yokozeki W, Koseki H, Hirabayashi Y, Gotoh Y. 2018. Ubiquitination-independent repression of PRC1 targets during neuronal fate restriction in the developing mouse neocortex. Dev Cell 47: 758-772.e5. doi:10.1016/j.devcel .2018.11.018

Wang Y, Lin L, Lai H, Parada LF, Lei L. 2013. Transcription factor Sox1 1 is essential for both embryonic and adult neurogenesis. Dev Dyn 242: 638-653. doi:10.1002/dvdy.23962

Zechner D, Müller T, Wende H, Walther I, Taketo MM, Crenshaw EB III, Treier M, Birchmeier W, Birchmeier C. 2007. Bmp and Wnt/ $\beta$-catenin signals control expression of the transcription factor Olig3 and the specification of spinal cord neurons. Dev Biol 303: 181-190. doi:10.1016/j.ydbio.2006.10.045

Zhang Y, Chen K, Sloan SA, Bennett ML, Scholze AR, O'Keeffe S, Phatnani HP, Guarnieri P, Caneda C, Ruderisch N, et al. 2014. An RNA-sequencing transcriptome and splicing database of glia, neurons, and vascular cells of the cerebral cortex. I Neurosci 34: 11929-11947. doi:10.1523/JNEUROSCI.186014.2014 


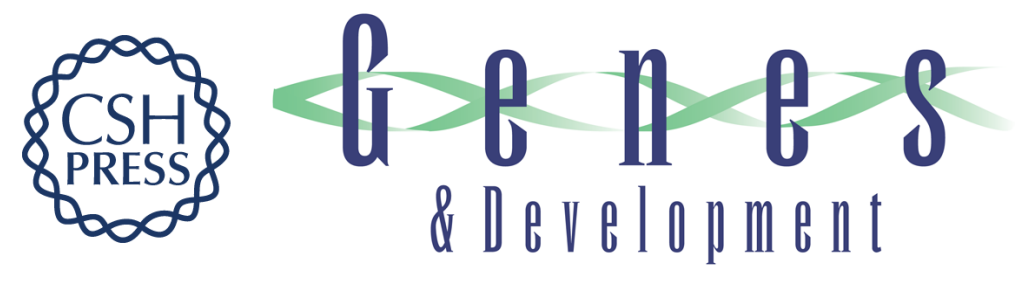

\section{Neural stem/precursor cells dynamically change their epigenetic landscape to differentially respond to BMP signaling for fate switching during brain development}

Sayako Katada, Jun Takouda, Takumi Nakagawa, et al.

Genes Dev. 2021, 35: originally published online October 21, 2021

Access the most recent version at doi:10.1101/gad.348797.121

Supplemental Material

References

Creative

Commons

License

Email Alerting

Service
http://genesdev.cshlp.org/content/suppl/2021/10/21/gad.348797.121.DC1

This article cites 60 articles, 21 of which can be accessed free at: http://genesdev.cshlp.org/content/35/21-22/1431.full.html\#ref-list-1

This article, published in Genes \& Development, is available under a Creative Commons License (Attribution-NonCommercial 4.0 International), as described at http://creativecommons.org/licenses/by-nc/4.0/.

Receive free email alerts when new articles cite this article - sign up in the box at the top right corner of the article or click here.

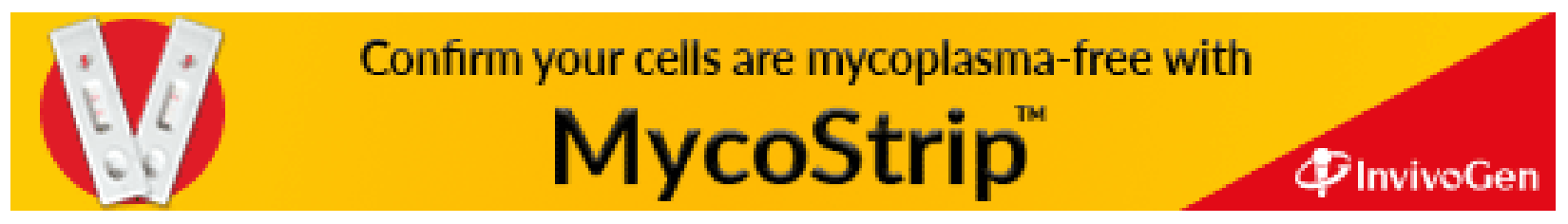

